\title{
Regular Strongly Compactness and Regular Strongly Connectedness in Topological Space
}

\author{
${ }^{1}$ R. Rajeswari, ${ }^{2}$ S. Darathi, ${ }^{3}$ D. Deva Margaret Helen \\ ${ }^{1}$ Assistant professor in Mathematics, \\ ${ }^{2,3}$ Masters in Mathematics \\ ${ }^{1,2,3}$ Thassim Beevi Abdul Kader College for Women \\ Kilakarai, Ramanathapuram.
}

\begin{abstract}
In this paper we proved that the regular strongly separated sets in the topological space.Also we extend our work in regular strongly compactness and connectness in the topological space.

\section{INTRODUCTION}

M.Karpagadevi and A.Pushpalatha already proved the RW-Closed maps and RW- Open maps in topological spaces later they worked on RW-continuous map and RW-irresolute maps in the topological space. M.Karpagadevi and A.Pushpalatha already proved that regular weakly separated sets in the topological space. We introduce the structure of regular strongly separated

sets.Also we give the concept of regular strongly compactness and regular strongly connectedness in the topological space.
\end{abstract}

\subsection{Definition:}

\section{PRELIMINARIES}

In a topological space $\mathrm{X}$ is called regular space if every closed subset $\mathrm{C}$ of $\mathrm{X}$ and the point $\mathrm{p}$ not contained in $\mathrm{C}$ admit non overlapping open neighourhoods. Thus $\mathrm{p}$ and $\mathrm{C}$ can be separated by neighbourhoods this is known as regular space.

\subsection{Definition:}

A topological space $\mathrm{X}$ is called separated if given any two distinct points $\mathrm{x}$ and $\mathrm{y}$ are disjoint by neighourhoods.

\subsection{Definition:}

A topological space $\mathrm{X}$ is called compact if every open cover has a finite subcover that covers $\mathrm{X}$.

\subsection{Definition:}

A topological space $\mathrm{X}$ is called complete if every Cauchy sequence has converges in $\mathrm{X}$.

\subsection{Definition:}

The weak topology on $\mathrm{X}$ is the initial topology with respect to dual space family $\mathrm{X}^{*}$.It is also known as Coarsest topology such that each element of $\mathrm{X}^{*}$ remains the continuous function and this is differ from ordinary topology that ordinary topology is called strong topology.

\subsection{Definition:}

Let $(\mathrm{X}, \tau)$ be a topological space, two non empty subsets A and B are said to be weakly separated if $\mathrm{A} \cap r w \mathrm{cl}(\mathrm{B})=\varnothing$ and $\mathrm{rw}-$ $\operatorname{cl}(\mathrm{A}) \cap B=\emptyset$

\subsection{Analogy:}

Two regular weakly separated sets are always disjoint.

\subsection{Definition}

A collection $\left\{A_{i}: i \in I\right\}$ of regular weakly open sets in a topological space $X$ is called a rw-open cover of a subset $B$ in $X$ if $B \subseteq$ i $\cup\left\{A_{i}: i \in I\right\}$

\subsection{Definition:}

A topological space $\mathrm{X}$ is regular weakly compact if every rw-open cover of $\mathrm{X}$ has finite sub cover of $\mathrm{X}$.

\subsection{Definition:}

A subset $B$ of a topological space $X$ is called rw-compact relative to $X$ if for every collection $\left\{A_{i}: i \in I\right\}$ of rw-open subsets of $X$ such that $B \subseteq{ }_{i \in I} \cup\{A i: i \in I\}$ there exist a finite subset $I_{0}$ of $I$ such that $B \subseteq \subseteq_{i \in I o} \cup\left\{A_{i}: i \in I_{0}\right\}$. 


\subsection{Definition:}

A subset $\mathrm{B}$ of a topological space $\mathrm{X}$ is called rw-compact if $\mathrm{B}$ is rw-compact as the subspace of $\mathrm{X}$.

\subsection{Definition:}

A topological space $\mathrm{X}$ is regular weakly connected if $\mathrm{X}$ cannot be written as a union of two disjoint non empty rw-open sets.

\section{MAIN RESULT}

\subsection{Definition:}

Let $(\mathrm{X}, \tau)$ be a topological space, two non empty subsets $\mathrm{A}$ and $\mathrm{B}$ are said to be strongly separated if there exist an open sets $\mathrm{U}$ and $\mathrm{V}$ such that $\mathrm{A} \subset \mathrm{U} ; \mathrm{B} \subset \mathrm{V}$ and $\mathrm{U} \cap \mathrm{V}=\varnothing$.

\subsection{Theorem:}

Two regular strongly separated sets are always disjoint.

\section{Proof:}

Let $\mathrm{A}$ and $\mathrm{B}$ be regular strongly sets,by the 3.1 definition there exist an openset $\mathrm{U}$ and $\mathrm{V}$ such that $\mathrm{A} \subset \mathrm{U} ; \mathrm{B} \subset \mathrm{V}$ and $\mathrm{U} \cap \mathrm{V}=\emptyset$.This implies that $\mathrm{A} \cap \mathrm{B}=\varnothing$.Hence $\mathrm{A}$ and $\mathrm{B}$ are disjoint.

\subsection{Example:}

If $X=\{a, b, c, d, e, f, g, h\}, \tau=\{X, \varnothing,\{c, d\},\{d, f\},\{a, e\},\{a, g\},\{b, c, d, e\},\{a, c, d\}\}, U=\{c, d, f\}$ then the interior is $U^{0}=\{c, d, f\}$ so $U$ is an open set,let $\mathrm{A}=\{\mathrm{c}, \mathrm{d}\}[\mathrm{A} \subseteq U]$ and $\mathrm{V}^{0}=\{\mathrm{a}, \mathrm{e}, \mathrm{g}\}$ so $\mathrm{V}$ is an open set,let $\mathrm{B}=\{\mathrm{a}, \mathrm{e}\}[\mathrm{B} \subseteq \mathrm{V}]$ if $\mathrm{U} \cap \mathrm{V}=\emptyset$ then $\mathrm{A} \cap \mathrm{B}=\emptyset$.

\subsection{Theorem:}

Let $\mathrm{A}$ and $\mathrm{B}$ be two regular strongly separated sets of $(\mathrm{X}, \tau)$.If $\mathrm{C} \subseteq \mathrm{A}$ and $\mathrm{D} \subseteq \mathrm{B}$ then $\mathrm{C}$ and $\mathrm{D}$ are regular strongly separated.

Proof:

Let $\mathrm{A}$ and $\mathrm{B}$ be regular strongly separated sets of a topological space $(\mathrm{X}, \tau)$ then by the definition there exist the open sets $\mathrm{U}, \mathrm{V}$ such that $\mathrm{A} \subset \mathrm{U}, \mathrm{B} \subset \mathrm{V}$

$\mathrm{U} \cap \mathrm{V}=\varnothing$.let $\mathrm{C} \subseteq \mathrm{A}$ and $\mathrm{D} \subseteq \mathrm{B}$ then we have two open set $\mathrm{U}, \mathrm{V}$ then $\mathrm{C} \subset \mathrm{U}, \mathrm{D} \subset V, \mathrm{U} \cap \mathrm{V}=\varnothing$ thus $\mathrm{C}$ and $\mathrm{D}$ are regular strongly separated.

\subsection{Example:}

We have to follow 3.3 example let $\mathrm{C}=\{\mathrm{d}\} \subseteq A \subset U, \mathrm{D}=\{\mathrm{e}\} \subseteq B \subset V$ it follows that $\mathrm{C} \subset \mathrm{U}, \mathrm{D} \subset V, \mathrm{U} \cap \mathrm{V}=\varnothing$ thus $\mathrm{C}$ and $\mathrm{D}$ are regular strongly separated.

\subsection{Theorem:}

Two regular strongly closed subset of a topological space $(\mathrm{X}, \tau)$ are rs-separated iff they are disjoint.

Proof:

If rs-separatedd sets are disjoint then rs closed separated sets are disjoint.

Conversely,

Let $\mathrm{A}$ and $\mathrm{B}$ be two disjoint rs closed sets,since $\mathrm{A} \subset \mathrm{U}, \mathrm{B} \subset \mathrm{V}$ then we have rs-cl $(\mathrm{A})=\mathrm{A}, \mathrm{rs}-\mathrm{cl}(\mathrm{B})=\mathrm{B}$ and $\mathrm{A} \cap \mathrm{B}=\varnothing$ this implies that $\mathrm{U} \cap V=\emptyset$.consequently $\mathrm{A} \cap r s-\mathrm{cl}(\mathrm{B})=\varnothing$ and $\operatorname{rs}-\mathrm{cl}(\mathrm{A}) \cap B=\emptyset$ hence $\mathrm{A}$ and $\mathrm{B}$ are rs-separated.

\section{7 lemma:}

If the union of two rs-separated sets in a rs-closed set then the individual sets are rs-closures are rs-closures of themselves.

\subsection{Theorem:}

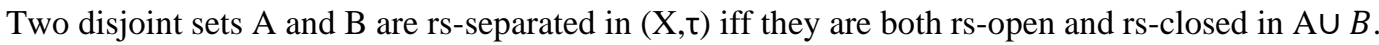

Proof:

Two disjoint sets $\mathrm{A}$ and $\mathrm{B}$ are rs-separated in $(\mathrm{X}, \tau)$ then $\mathrm{A} \cap r s-\mathrm{cl}(\mathrm{B})=\varnothing$ and rs-cl( $\mathrm{A}) \cap B=\emptyset$.let $\mathrm{R}=\mathrm{A} \cup B$ then rs-cl $\mathrm{R}(\mathrm{A})=\mathrm{rs}-$ $\operatorname{cl}(\mathrm{A}) \cap R=\operatorname{rscl}(A) \cap(A \cup B)=A$ therefore $\mathrm{A}$ is rs-closed in $\mathrm{R}$ similarly $\mathrm{B}$ is rs-closed inR.now $\mathrm{A} \cap B=\emptyset$ and $A \cup B=R$ so $\mathrm{A}$ and $\mathrm{B}$ are complements of each other in $\mathrm{R}$ thus each one of $\mathrm{A}$ and $\mathrm{B}$ is rs-open in $\mathrm{R}$.

Conversely,let $\mathrm{A}$ and $\mathrm{B}$ be disjoint sets which are both rs-open and rs-closed in $\mathrm{R}=\mathrm{A} \cup B$ then $A=\operatorname{rs}_{-\mathrm{cl}}(\mathrm{A})=\mathrm{rs}-\mathrm{cl}(\mathrm{A}) \cap R=$ $\operatorname{rscl}(A) \cap(A \cup B)=\mathrm{A} \cup[\operatorname{rscl}(A) \cap B]$ but $\mathrm{A} \cap B=\emptyset$ this implies $\mathrm{A} \cap[\mathrm{rs}-\mathrm{cl}(\mathrm{A}) \cap B]=\emptyset$. Now $\mathrm{A}$ and $[\mathrm{rs}-\mathrm{cl}(\mathrm{A}) \cap B]$ are disjoint

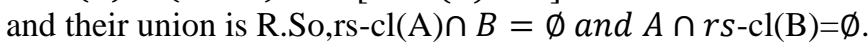

Hence A and B are rs-separated.

\subsection{Definition:}

A collection $\left\{A_{i}: i \in I\right\}$ of regular strongly open sets in a topological space $X$ is called a rs-open cover of a subset $B$ in $X$ if $B \subseteq$ iєI $\mathrm{U}\left\{\mathrm{A}_{\mathrm{i}}: \mathrm{i} \in \mathrm{I}\right\}$

\subsection{Definition:}


A topological space $\mathrm{X}$ is regular strongly compact if every rs-open cover of $\mathrm{X}$ has finite sub cover of $\mathrm{X}$.

\subsection{Definition:}

A topological space is said to be strongly compact if every cover of pre open sets admits a finite sub cover.

\subsection{Theorem:}

A rs-closed subset of rs-compact space is rs-compact relative to $\mathrm{X}$.

\section{Proof:}

Let A be a rs-closed subset of a rs-compact space X. Then Ac is rs-open in X. Let $\mathrm{S}$ be a rs-open cover of $\mathrm{A}$ in $\mathrm{X}$. Then, $\mathrm{S}$ along with $A c$ forms a rs-open cover of $X$. Since $X$ is rs-compact, it has a finite sub cover say $\left\{G_{1}, G_{2}, \ldots . . . G n\right\}$. If this subcover contains $\mathrm{A}_{\mathrm{c}}$, we discard it. Otherwise leave the subcover as it is. Thus we have obtained a finite subcover of A and so A is rs-compact relative to $\mathrm{X}$.

\subsection{Theorem:}

A closed subset of rs-compact space is rs-compact relative to X.

\section{Proof :}

Let A be a closed subset of a rs-compact space X. Then A is rs-closed. Therefore by Theorem 3.5,every rs-closed subset of a rscompact space is rs-compact relative to X. Hence A is rs-compact space related to X.

\subsection{Definition :}

A topological space $(\mathrm{X}, \tau)$ is said to be regular strongly connected (briefly rs-connected) if $\mathrm{X}$ cannot be written as a union of two disjoint non-empty rs-open sets.

\subsection{Theorem:}

Every rw-connected space is connected .

\section{Proof:}

Let $(X, \tau)$ be an rs-connected space. Suppose that $X$ is not connected. Then $X=A \cup B$, where A and B are disjoint non-empty open sets in $(X, \tau)$. Since every open set is rs-open, A and B are rs-open. Therefore, $X=A \cup B$, where A and B are disjoint nonempty rs-open sets in $(X, \tau)$. This contradicts the fact that $\mathrm{X}$ is rs-connected and so $\mathrm{X}$ is connected.

\subsection{Theorem:}

A topological space $(\mathrm{X}, \tau)$ is rs-disconnected if there exists a non-empty proper subset of $\mathrm{X}$ which is both rs-open and rs-closed.

\section{Proof:}

Let $\mathrm{A}$ be a non-empty proper subset of $\mathrm{X}$ which is both rs-open and rs-closed. Then clearly $\mathrm{A}_{\mathrm{c}}$ is a non-empty proper subset of $X$ which is both rs-open and rs-closed. Thus $A \cap A_{c}=\phi$ and therefore $A \cap r s-c l\left(A_{c}\right)=\phi$ and rs-cl $\left(A_{c}\right) \cap A=\phi$. Also, $X=A \cup A_{c}$. Thus $\mathrm{X}$ is the union of two non-empty rs-separated sets. Hence $\mathrm{X}$ is rs-disconnected.

\subsection{Theorem:}

If the sets $\mathrm{A}$ and $\mathrm{B}$ form an rs-separation of $(\mathrm{X}, \tau)$ and if $(\mathrm{Y}, \sigma)$ is an rs-connected subspace of $(\mathrm{X}, \tau)$ the $\mathrm{Y}$ lies entirely within either A or B.

\section{Proof:}

Since $\mathrm{A}$ and $\mathrm{B}$ are both rs-open in $\mathrm{X}$, the sets $\mathrm{A} \cap \mathrm{Y}$ and $\mathrm{B} \cap$ Yare rs-open in $\mathrm{Y}$. These two sets are disjoint and their union is $\mathrm{Y}$. If they were both non-empty, they would constitute an rs-separation of $Y$. Therefore one of them is empty. Hence $Y$ must lie entirely in A or in B.

\subsection{Theorem:}

If every two points of a set A are contained in some rs-connected subset of A, then A is rs-connected.

\section{Proof :}

Suppose that A is not rs-connected. Then $A$ is the union of two non-empty disjoint rs-open sets $G$ and $H$. Let $g \in G$ and $h \in H$. Then $\mathrm{g}$ and $\mathrm{h}$ are two distinct points of A. By hypothesis, there exists a rs-connected subset $\mathrm{B}$ of $\mathrm{A}$ such that $\mathrm{g}, \mathrm{h} \in \mathrm{B}$. But $\mathrm{B}$ is an rs-connected subset of a rs-disconnected set $A$, we have $B \subseteq G$ or $B \subseteq H$. Since $G$ and $H$ are disjoint and $B$ contains atleast one point of $\mathrm{G}$ and one of that of $\mathrm{H}$. This is a contradiction. Hence $\mathrm{A}$ is rs-connected. 


\section{REFERENCE}

[1] Karpagadevi. M and Pushpalatha. A., "RW-Continuous maps and RW- irresolute maps in topological spaces", International Journal Of Mathematics Trends and Technology,Vol.4,Issue 2-2013,21-25.

[2] Karpagadevi. M and Pushpalatha. A., "RW-Closed maps and RW- Open maps in topological spaces", International Journal Of Computer Applications Technology and Research,Vol.2,Issue 2-2013,91-93.

[3] Benchalli.S .S and Wali. R.S On R $\omega$-closed sets in topological spaces, Bull. Malays. Math. Sci. soc(2) 30(2) (2007), 99-110.

[4] Dr Maio.G. and Noiri, T., On s-closed spaces, Indian J. Pure APPI. Math., 18(3) (1987), 226-223. 